\title{
Measurement of the bone endocortical region using clinical CT
}

\author{
R.A. Pearson ${ }^{\mathrm{a}, *}$, G.M. Treece ${ }^{\mathrm{a}}$ \\ ${ }^{a}$ University of Cambridge, Department of Engineering, Trumpington Street, Cambridge, CB2 1PZ, UK
}

\begin{abstract}
The extent of the endocortical region and cortical bone mineral density (cBMD) throughout the proximal femur are of interest as both have been linked to fracture risk and osteoporosis treatment response. Non-invasive in-vivo clinical CT-based techniques capable of measuring the cortical bone attributes of thickness, density and mass over a bone surface have already been proposed. Several studies have robustly shown these methods to be capable of producing cortical thickness measurements to a sub-millimetre accuracy. Unfortunately, these methods are unable to provide high quality cBMD estimates, and are not designed to measure any attributes over the endocortical region of cortical bone. In this paper, we develop a cortical bone mapping based technique capable of providing an improved cBMD estimate and a measure of the endocortical width, while maintaining similar quality cortical thickness and trabecular bone mineral density (tBMD) estimates. The performance of the technique was assessed using a paired dataset of ex-vivo QCT and HR-pQCT scans across 72 proximal femurs. The HR-pQCT scans were analysed using a new method developed for this study: high resolution tissue classification (HRTC). In HRTC the cortical, endocortical and sub-surface trabecular bone features are extracted from the partially resolvable microarchitectural details in the HR-pQCT scan. We demonstrate that measurement of the endocortical extent from QCT is possible with an accuracy of $-0.15 \pm 0.71 \mathrm{~mm}$, and that local cBMD can be measured down to densities of $300 \mathrm{mg} / \mathrm{cm}^{3}$.
\end{abstract}

Keywords: computed tomography, endocortical region, bone mineral density, cortical thickness

\section{Introduction}

Fragility fractures due to osteoporosis and other skeletal diseases are a significant public health burden (Kanis et al. 2013; World Health Organisation, 2007), which is

5 set to increase as the global population continues to age. The societal cost of hip fractures is particularly severe due to high morbidity and mortality associated with this fracture (World Health Organisation, 2007). The quantity and severity of fragility fractures can be reduced through early

10 identification and treatment of those at risk. Fracture risk prediction, treatment assessment and the monitoring of ${ }_{35}$ osteoporosis disease progression in the proximal femur are hence all of particular interest.

In current clinical practice, areal bone mineral den15 sity (aBMD), a two dimensional measure of bone mineral density extracted from Dual-energy X-ray Absorptiometry ${ }_{40}$ (DXA) scans, is used to assess fracture risk and treatment effectiveness (World Health Organisation, 2007). Despite widespread use, DXA scans are not capable of directly easuring three dimensional features of bone (Ohnaru et al. 2013) or focal variations in bone structure, both of which have been linked to fracture risk (Holzer et al., 2009, Poole et al., 2012, Verhulp et al., 2008). Thus a three dimen-

\footnotetext{
*Corresponding author. Tel. +44 1223339707.

Email addresses: rpearson89@gmail.com (R.A. Pearson), gmt11@cam.ac.uk (G.M. Treece)
}

sional imaging modality combined with a technique capa25 ble of preserving and extracting these features is desirable. As most mineralised bone is either cortical or subsurface trabecular bone, this is the region of particular interest for modelling (Yang et al., 2012).

Quantitative Clinical CT (QCT) scans provide such a three dimensional measure of BMD but have limited resolution. Much of the cortex is a thin structure well below this resolution, and its measurement is challenging. Several techniques exist for measuring cortical and subsurface trabecular features from QCT (Hangartner and Gilsanz, 1996, Hangartner and Short, 2007, Streekstra et al., 2007. Treece et al., 2010). These techniques, however, are only marginally more effective than aBMD-based methods at fracture risk prediction and treatment assessment (Poole et al., 2012, Treece et al., 2015, Yang et al., 2012). Currently, the most accurate are model-based techniques. These use a transect of cortical and subsurface trabecular bone sampled from the QCT scan, a parametrised estimate of the underlying density, and an estimation of the imaging blur, to model the underlying cortical features using deconvolution.

Cortical Bone mapping (CBM) is one of these modelbased techniques. Many local measurements are made over the surface of the bone and combined to provide surface maps of the measured features. This allows foso cal regional variations between populations to be detected. Previous implementations used a simple three tier model 
to represent the density in profile across the cortex at each surface location (Treece and Gee, 2015, Treece et al. 110 2010, 2012). This three tier model is only capable of meadensity (cBMD) and the trabecular bone mineral density (tBMD), where these measurements are single aggregate values across the entire cortical or trabecular re-115 gion included in the profile. These values can be used 60 to calculate the mass surface density (MSD), which is the mass of mineralised cortical bone per unit area over the periosteal surface (Treece et al., 2012). MSD is more resistant to errors resulting from the limited resolution of QCT (Treece et al., 2012). However, the CBM model

65 does not measure the endocortical region, which exists be- ${ }_{120}$ tween the compact cortical bone and the porous trabecular bone (Ma, 2014). It has been suggested that this is a region of elevated remodelling in later life (Seeman and Delmas, 2006, Zebaze et al. 2013). As such, it may pro-

70 vide an early indication of deteriorating bone quality, and a measure of targeted regeneration caused by therapies for ${ }^{125}$ the treatment of osteoporosis.

The initial implementation of CBM used a single fixed cBMD value applied over the measurement surface: this

75 increased the stability and precision of the technique (Treece et al. 2010, 2012). In addition to not providing any local ${ }^{30}$ cBMD measurement, it also limited the accuracy of the model at any location where the cortical density varied from the global estimate, and increased the bias of corti-

${ }_{80}$ cal thickness for thin structures (Treece and Gee, 2015). For these reasons, a two phase model-based fitting technique termed CBMv2, aimed at relaxing the rigidity of the cBMD constraint, was proposed (Treece and Gee, 2015). Although this technique enabled local cBMD measure-

85 ments, while improving the overall stability of the method and improving the quality of the cortical thickness mea- ${ }^{140}$ surements, the quality of the cBMD measurements remained poor (Treece and Gee, 2015).

cBMD is affected by both the degree of mineralisation of bone (DMB) and porosity of cortical bone (Augat and Schorlemmer, 2006, Boivin et al., 2008; Follet et al., 2004, McCalden et al., 1993). The DMB is an intrinsic material property of bone tissue, while porosity is a property of the microarchitecture of bone. cBMD increases with DMB

95 but decreases with porosity, while fracture risk decreases with DMB and increases with porosity. Hence a decrease ${ }^{150}$ in cBMD due to either mechanism increases fracture risk. The relative contributions of each to cBMD cannot be distinguished at the comparatively low resolution of QCT.

100 Instead it is only possible to measure the average cBMD, which is still a significant indicator of bone strength and ${ }^{155}$ fracture risk (Augat and Schorlemmer, 2006, Follet et al. 2004 , McCalden et al., 1993).

The aim of this work is to extend the CBM technique to both measure the thickness of the endocortical region for the first time from QCT scans, and to more accurately measure the cBMD. In Section 2 the current CBM technique is detailed before a more representative model of the cortical and subsurface trabecular region, and a new variable cBMD fitting scheme, are introduced. In Section 3 the quality of the endocortical thickness and cBMD measures are assessed, against equivalent measures extracted from HR-pQCT scans, using a dataset of paired QCT and HR-pQCT scans. In Section 4 the ability of the new model and fitting scheme to measure the cortical and subsurface structure of bone is examined. Conclusions on the suitability of the proposed model and fitting scheme are then drawn in Section 5.

\section{Methods}

\subsection{Constrained three-tier rectangle model}

$\mathrm{CBM}$ is a pipeline-based technique, as shown in Figure 1, in which many features are measured at different locations over a surface and combined into surface maps of each feature. Each set of feature measurements is extracted from a parametrised model that describes the density variation along a transect through cortical and subsurface trabecular bone at a particular surface location. Previous work has used the three-tier rectangle model, termed the 'rectangle model' throughout the remainder of this paper, shown in Figure 2A. The model has instantaneous transitions between the different tissues and a constant density over the entire cortical cross-section. It is also constrained at each surface location to share the same globally fixed cBMD value. The remaining model features and the image point spread function (PSF) are then varied, until the resulting density profile estimate best matches the density profile sampled from the image. This is achieved with the Levenberg-Marquardt optimisation method (LMM) as discussed in Section 2.3. The resulting models can be used to generate cortical thickness and tBMD surface maps.

A key component of this algorithm is the convolution of the density model with the image PSF, which is presumed to be Gaussian, to produce the density profile estimate. Convolution is an $\mathcal{O}\left(N^{2}\right)$ operation. This can be reduced to an order $\mathcal{O}(N)$ integration by first differentiating Eqn 1a and then applying the 'Sifting' property of the subsequent Dirac Delta functions as shown in Eqn 1c (Treece et al. 2012), where $y_{s t}, y_{c b}, y_{t b}, x_{P}, x_{E}$ and $\sigma$ respectively correspond to the density of soft tissue, the density of cortical bone, the density of trabecular bone, the location of the cortex periosteal edge, the location of the cortex endosteal edge, and the standard deviation of the Gaussian PSF. $H(x)$ represents the unit step function, and $\operatorname{erf}()$ is the error function that results from integrating a 


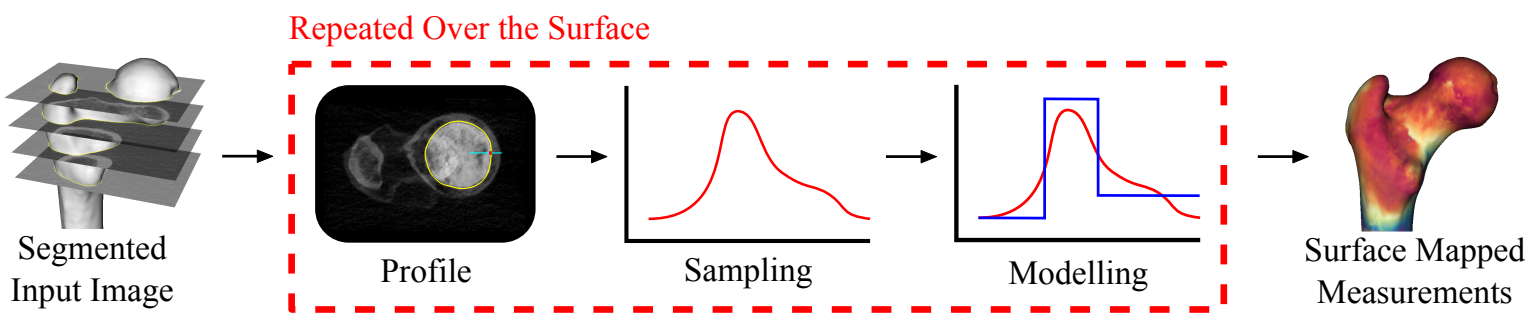

Figure 1: The Cortical Bone Mapping Pipeline.

Gaussian.

$$
\begin{aligned}
y(x)= & y_{s t}+\left(y_{c b}-y_{s t}\right) \cdot H\left(x-x_{P}\right) \\
& +\left(y_{t b}-y_{c b}\right) \cdot H\left(x-x_{E}\right) \\
g(x)= & \frac{1}{\sqrt{2} \pi \sigma} e^{-\frac{-x^{2}}{2 \sigma^{2}}} \\
y_{\text {blur }}(x)= & y(x) * g(x) \\
= & \frac{y_{s t}+y_{t b}}{2}+\frac{y_{c b}-y_{s t}}{2} \operatorname{erf}\left(\frac{x-x_{P}}{\sigma \sqrt{2}}\right) \\
& +\frac{y_{t b}-y_{c b}}{2} \operatorname{erf}\left(\frac{x-x_{E}}{\sigma \sqrt{2}}\right)
\end{aligned}
$$

The model-fitting problem is ill-posed due to the large number of model parameters relative to the information available in the blurred density profile. This is the reason for using the simple rectangle model, and constraining the cortical density to reduce the number of unconstrained parameters to five. The recently proposed CBMv2 method relaxes the rigidity of the cBMD constraint by performing two CBM measurement steps: the first with the global cBMD constraint, and the second with a locally adjusted ${ }_{19}$ cBMD constraint based upon the difference between the local blur estimate from the initial CBM measurement and a global estimate of the PSF blur (Treece and Gee, 2015). This allows for some variability in the cBMD, while maintaining the number of unconstrained parameters at five. ${ }^{195}$

\subsection{Endocortical region model}

The endocortical region is the interface between the cortical and trabecular bone. It is of particular interest as it has been suggested that this is the primary site of emodelling in later life, when most fragility fractures occur (Seeman and Delmas, 2006, Zebaze et al., 2013). The increased endocortical remodelling has been linked to a decrease in the DMB and also an increase in porosity (Boivin et al. 2008; Follet et al., 2004, Zebaze et al., 2013), both which result in decreased BMD over the endocortical region. We model this by adapting the rectangular model to include a finite width linear transition from 'dense' cortical bone to trabecular bone as shown in Figure 2 $\mathrm{B}$. This new model is called the 'endocortical model' throughout he rest of this paper.

Having effectively split the location of the endosteal edge in two, we can measure the 'dense' cBMD (dBMD),
A

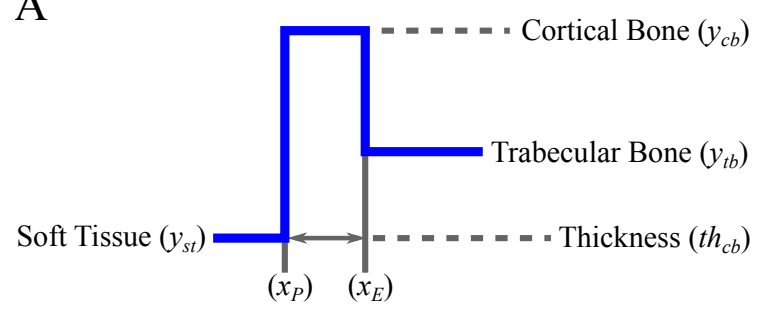

B

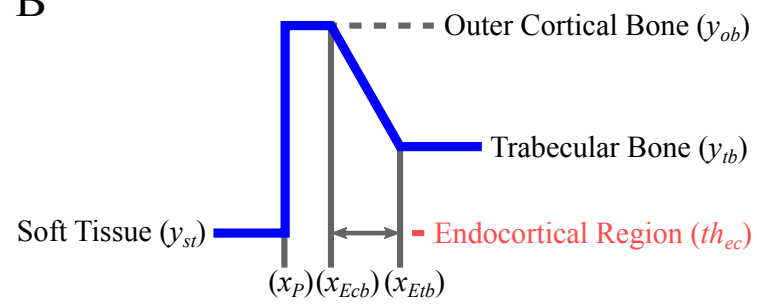

Figure 2: The parametrised bone surface density models. (A) The rectangle model. (B) The endocortical region model.

the dense cortical bone thickness, the width of the endocortical region and tBMD. These may also be combined to derive measures of the cortical bone thickness $t h_{c b}$ and cBMD. Eqn 2 is used to calculate these measurements by assuming the cortical bone region extends half-way through the endocortical region. In this and the following Eqn 3 the meaning of $y_{s t}, y_{t b}, x_{P}$, and $\sigma$ remain unchanged compared to Eqn 1a, while $y_{d b}$ replaces the $y_{c b}$ of the rectangle model and corresponds to dBMD. $x_{E c b}$ and $x_{E t b}$ correspond to the location of the beginning and end of the endocortical region.

$$
\begin{aligned}
& t h_{c b}=\quad\left(x_{E c b}-x_{P}\right)+\frac{1}{2}\left(x_{E t b}-x_{E c b}\right) \\
& y_{c b}=y_{d b} \frac{x_{E c b}-x_{P}}{t h_{c b}}+\left(\frac{3}{4} y_{d b}+\frac{1}{4} y_{t b}\right) \frac{\frac{1}{2}\left(x_{E t b}-x_{E c b}\right)}{t h_{c b}}(2 \mathrm{~b})
\end{aligned}
$$

Once again, it is desirable to avoid performing an $\mathcal{O}\left(N^{2}\right)$ convolution at each step in the optimisation process. The presence in the model of $R(x)$, the Ramp function, requires an additional integration-differentiation step before the 'Sifting' property can be applied to the resulting Dirac Delta functions, producing the more complex result in Eqn 3. The required integral of the error function is not supported in standard libraries, however it can be precomputed and accessed at runtime using a look-up table. 


$$
\begin{aligned}
y(x)= & y_{s t}+\left(y_{d b}-y_{s t}\right) H\left(x-x_{P}\right) \\
& +\frac{y_{t b}-y_{d b}}{x_{E t b}-x_{E c b}} R\left(x-x_{E c b}\right) \\
& -\frac{y_{t b}-y_{d b}}{x_{E t b}-x_{E c b}} R\left(x-x_{E t b}\right) \\
y_{\text {blur }}(x)= & y(x) * g(x) \\
= & \frac{y_{s t}+y_{t b}}{2}+\frac{y_{d b}-y_{s t}}{2} \operatorname{erf}\left(\frac{x-x_{P}}{\sigma \sqrt{2}}\right) \\
& +\frac{\left(y_{t b}-y_{d b}\right)}{\left(x_{E t b}-x_{E c b}\right)} \int_{-\infty}^{x} \operatorname{erf}\left(\frac{\xi-x_{E c b}}{\sigma \sqrt{2}}\right) d \xi \\
& -\frac{\left(y_{t b}-y_{d b}\right)}{\left(x_{E t b}-x_{E c b}\right)} \int_{-\infty}^{x} \operatorname{erf}\left(\frac{\xi-x_{E t b}}{\sigma \sqrt{2}}\right) d \xi
\end{aligned}
$$

The LMM is also used to fit the endocortical model. As with the constrained rectangle model, the $\mathrm{dBMD}$ value can be fixed before performing CBM to increase the stability of the optimiser. However, without any other changes to the overall CBM process, the stability will be lower than the rectangle CBMv2 method, due to the inclusion of the additional endocortical width parameter that further destabilises the already ill-posed problem. This can ${ }^{250}$ be counteracted, to some extent, through the careful management of the optimisation process.

\subsection{CBM optimisation}

In the CBM method an optimiser is used to select those ${ }^{255}$ model parameters which give the best possible alignment with the sampled image densities. This is a non-linear least-squares problem, in which the squared error between the sampled image density and the blurred parametrised model is minimised, by iterative improvement of an initial ${ }^{260}$ guess, using the LMM (Moré, 1978). Crucial to this process is a problem-specific cost function which, when provided with the model parameters, updates an error vector containing differences between the modelled and sampled densities, as well as some other constraints relating to the ${ }^{265}$ physical validity of the model. It is this error vector which is minimised by the LMM.

\subsubsection{Validity of model parameters}

A density model is physically invalid if it has a nega- ${ }^{270}$ tive cortical thickness, a cBMD less than the tBMD, or a negative or extremely large Gaussian blur. The endocortical model is also invalid if it has a negative endocortical thickness. The error vector includes an element for each possible physically unrealistic trait; Eqn 4 is used to de- ${ }^{275}$ fine the error associated with each such trait. $\epsilon$ is the error reported in the error vector; $k$ is the scaling factor associated with that trait; $t$ is the model trait; and $t_{\text {minimum }}$ is the model trait value below which the model is physically invalid. The error value approaches 0 for valid traits, and exponentially increases with the magnitude of an invalid ${ }^{280}$

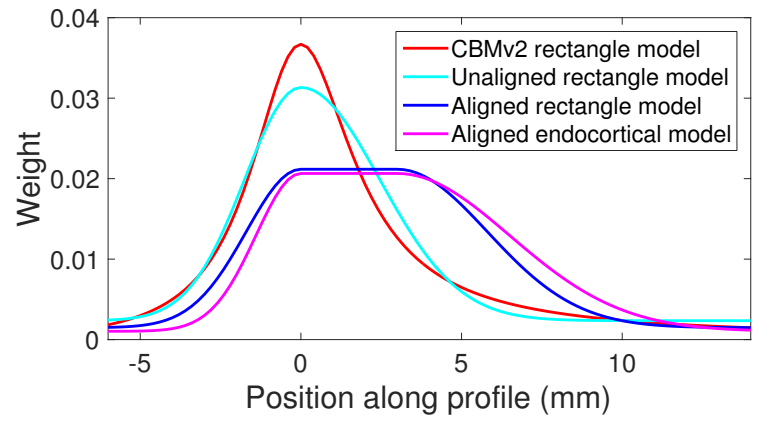

Figure 3: The different weighting functions used when calculating the cost function error vector during each optimisation stage in the CBM methods.

trait. $k$ is chosen to produce similar magnitude errors from each model trait.

$$
\epsilon=e^{k \cdot\left(t-t_{\text {minimum }}\right)}
$$

\subsubsection{Optimiser weighting function}

The error vector also includes an element for the signed difference between each sampled density value and the blurred model. These error values are scaled by a weighting function, which was used in previous implementations of the CBM method to encourage the optimiser to converge on a model that was closely aligned to the underlying periosteal surface and hence improve the model fit (Treece et al., 2010 As such, the weighting function controls the relative importance assigned to discrepancies between the blurred model and the sampled image density along the profile, with a tighter fit expected over portions of the profile assigned a higher relative weight. This means that the shape of the weighting function can be used to improve the quality of specific model traits, which has lead to the use of several different weighting functions as shown in Figure 3 .

The rectangular CBMv2 method uses the 'CBMv2 rectangle model' weighting function, whose peak is aligned with the initial estimate of the periosteal edge, i.e. the location of the initial surface segmentation. As previously described, in the first LMM optimisation, all other model values are initialised to the same generic constants for all locations. The results of this optimisation are used to initialise the second LMM, having re-aligned the weighting function to the updated periosteal edge estimate. The third LMM optimisation then makes use of the new cBMD constraint, adjusted based upon the image blur. The remaining weighting functions included in Figure 3 are used in the optimisation of the endocortical model by the Smoothly spatially constrained CBM (SSC-CBM) method described in Section 2.4. Note that the repeated optimisation runs are very fast, since the initial model parameters are usually very close to the converged solution.

\subsection{Smoothly spatially constrained CBM (SSC-CBM)}

The reduced stability of the endocortical model compared to the rectangle model can be combated by improv- 
ing the quality of the dBMD estimate used to constrain the endocortical model and by appropriately penalising physalso clinically desirable as dBMD is linked to bone strength ${ }_{340}$ and fracture risk (Augat and Schorlemmer, 2006, Follet et al., 2004; McCalden et al., 1993). The pipeline shown in Figure 4 was developed to provide improved dBMD estivalues observed in the proximal femur of HR-pQCT scans.345

The pipeline contains two endocortical CBM measurement steps. The first is a partially constrained CBM method that is used to produce dBMD estimates with LMM optimisations of which the first two use the rectangle model to improve stability. The first LMM uses350 a generically initialised and dBMD-constrained rectangle model. These model parameters are used to initialise the second LMM, which is still constrained by the generic MD value but with an aligned weighting function. The resulting parameters initialise the final LMM, which is an entirely unconstrained endocortical model with a generic $c_{35}$ endocortical thickness. This partially constrained configuration has greater stability than using a fully uncondBMD surface map is noisy, so it was spatially smoothed using Gaussian distance-weighted precision smoothing. Thę $\sigma$ of the Gaussian distance filter was specified globally at $6 \mathrm{~mm}$, while the precision value was calculated locally from 310 estimates of the variance of the LMM model parameters. These smoothed locally-varying dBMD values were used as constraints in the second CBM measurement step.

The endocortical SSC-CBM method uses the 'unaligned' and 'aligned' weighting function, shown in Figure 3. The

315 'unaligned rectangle model' is used with generically initialised rectangle models to produce periosteal alignment of the model. The 'aligned rectangle model' weighting ${ }_{370}$ function is used with rectangle models that have been initialised with the results from a previous LMM optimisaion. The initial cortical thickness value is used to define the width of the uniformly weighted region of the weighting function. The 'aligned endocortical model' weighting function is similarly used with endocortical models initialised with the results from a previous LMM optimisation.

\section{3. Experiments and results}

\subsection{Data acquisition}

The quality of the measurements produced with the proposed methods was assessed using direct comparison between QCT and HR-pQCT scans from an ethically apsame dataset used in the initial assessment of the CBMv2 method (Treece and Gee, 2015). The left and right femurs of 18 females and 17 males were stripped of soft tissue, submerged in a saline solution, then vacuum packed to dividuals was 77 years (range 59-96 years). The QCT data

was acquired using a Brilliance $64^{1}$ scanner at $120 \mathrm{kV}$ with a pixel sizing of $0.33 \times 0.33 \times 1 \mathrm{~mm}^{3}$. The Hounsfield Units were converted to density using a QRM-BDC calibration phantom ${ }^{2}$ scanned with each specimen.

The reference HR-pQCT data was captured using an $\mathrm{XTremeCT}{ }^{3}$ scanner at $70 \mathrm{kV}$ with a pixel sizing of $0.082 \times$ $0.082 \times 0.082 \mathrm{~mm}^{3}$. The HR-pQCT images were converted to density using a linear calibration curve provided by the manufacturer combined with a quadratic correction for non-linearities in the HU to BMD relationship as detailed in Appendix A. This gave a total of 74 paired high and low-resolution data sets from 37 patients. Two scan pairs were excluded, one for high levels of ring artefact in the HR-pQCT scan, and one for a HR-pQCT scan imaged with a restricted field of view. Further details of this study have been published previously (Dall'Ara et al., 2013a b).

\subsection{High resolution tissue classifier (HRTC)}

HR-pQCT scans were used to produce 'gold standard' measurements for direct comparison with the results of the newly proposed CBM scheme. This was achieved with a new technique called the High Resolution Tissue Classifier (HRTC), which produces localised cortical thickness, endocortical thickness, cBMD and tBMD measurements over a periosteal surface. The HRTC technique was developed as no previously published techniques provide localised measurements of both the dense cortical, endocortical and trabecular regions (Burghardt et al., 2010; Hildebrand and Rüegsegger, 1997; Laib et al., 1997; Liu et al. 2010; Treece et al., 2010, 2012; Zebaze et al. 2014). However, measurements from the HRTC method are defined in-line with equivalent aspects from other published HRpQCT methods. HRTC has four stages as shown in Figure 5. sampling, thresholding, sectioning, and modelling of the different regions.

The HRTC uses many closely sampled parallel profiles to discriminate between microstructure features and the cortical and subsurface trabecular macrostructure features that are actually of interest here. The profiles are sampled over a uniform grid at the pixel resolution within a fixed radius centred about each surface measurement location. The density samples along each profile are then thresholded as either 'bone' or 'not bone' in the thresholding step in Figure 5. The thresholds used were selected to densities in each scan. This allows the percentage of the profiles passing through bone at each sample location to be recorded, revealing the cross-sectional tissue structure at each sample location along the profile. The percentage profile is used in the sectioning step of Figure 5 as this provides a more reliable measure of the cross sectional structure than either a single density profile as illustrated

\footnotetext{
${ }^{1}$ Philips, Germany

${ }^{2}$ QMR Gmbh Germany

${ }^{3}$ Scanco Medical AG Switzerland
} 


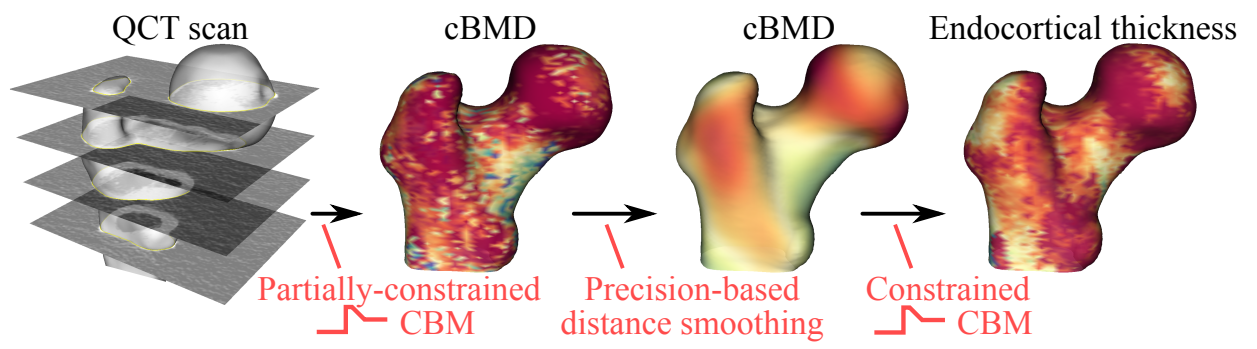

Figure 4: The Smooth spatially constrained CBM pipeline.

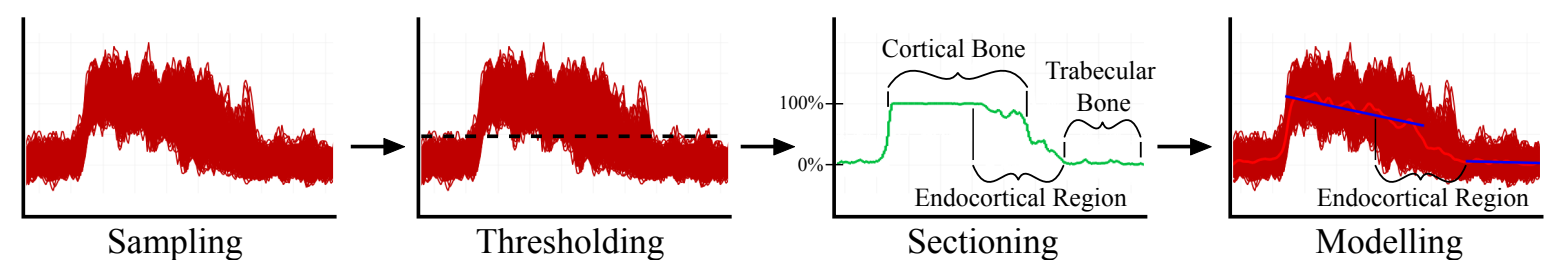

Figure 5: The four stages of the High Resolution Tissue Classifier method: sampling parallel profiles at the image resolution; thresholding each density sample into ossified or non-ossified tissue; sectioning the bone percentage profile to define the extents of the different tissues along its length; and modelling the density of each tissue type.

in Figure 6, or the mean cross-sectional density, which is 420 affected by the DMB of each profile in addition to the proportion of profiles passing though ossified and non-ossified tissue. The HR-pQCT scans were processed with parallel profiles sampled over a radius of $0.7 \mathrm{~mm}$ to match the QCT resolution as estimated based on the blur measured ${ }_{425}$ by the rectangular CBMv2 method. This gave a total of 225 parallel profiles at each measurement location.

The bone percentage is used to classify the profiles into four different regions: cortical bone, dense cortical bone, the endocortical region and trabecular bone. These regions ${ }_{430}$ were selected to allow the quality of both the rectangle and endocortical models to be assessed. Figure 5 shows the cortical, endocortical and trabecular regions: the dense cortical bone region lies between the start of the cortical bone and the start of the endocortical region. The cortical bone region extends from the first point where half the maximum percentage along the profile $\left(\%_{\max }\right)$ is reached, to the first point the percentage falls below the average of the $\%_{\max }$ and the mean trabecular percentage $\left(\%_{\bar{t} b}\right)$. This aims to represent the same region that is measured by the ${ }_{440}$ full-width half-max method used to validate previous implementations of the CBM method (Treece and Gee, 2015 Treece et al., 2010, 2012). The dense cortical bone region is the portion of the cortical bone before the first endocortical pore: defined as the point where the percentage ${ }_{445}$ array last falls below $\%_{\max }$, in line with previous methods (Zebaze et al., 2013). The endocortical region extends from the first endocortical pore in the cortical bone region to the first minimum after the percentage array drops to $\%_{\bar{t} b}$, and the trabecular region extends from the end of the endocortical region to the end of the profile.

\subsection{Experimental pipeline}

Figure 7 shows the pipeline used to evaluate the quality of the endocortical SSC-CBM method against both the HRTC validation method, and the previous 'best' implementation of the CBM: the rectangular CBMv2 method. First, a single canonical surface was used to segment each scan, as this allows for direct comparison between the measurements made on each paired set of QCT and HRpQCT scans, and between scans of different individuals. The canonical surface was registered to a manually segmented surface in each QCT scan using a locally affine free-form deformation calculated by an iterative closest point point registration algorithm (Feldmar and Ayache, 1996). A similarity registration was then applied between the registered QCT surface and a manually segmented surface in each matching HRpQCT scan. The rectangular CBMv2 and the endocortical SSC-CBM methods were applied to the QCT scans, while the HRTC validation technique was applied to the HRpQCT scans. Two iterations of mesh connectivity-based precision-weighted smoothing were performed over the CBM measurements, which were matched with two iterations of evenly weighted smoothing over the HRTC measurements. The CBM precisions were extracted from the estimated model parameter variances of the final LMM optimisation at each measurement site. The smoothing was performed to remove outlier measurements with very low precision optimisations. Figure 7 shows a representative example of the models produced by the endocortical SSC-CBM and HRTC methods at the same measurement location.

\subsection{Results}

The canonical surface contains 5580 measurement locations resulting in more than 400000 measurements across the 72 scans in the validation dataset. The quality of the 
A

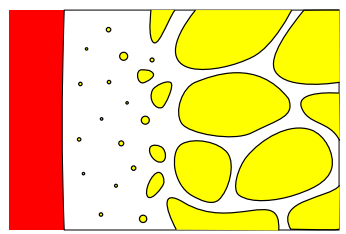

Soft Tissue

Mineralised Bone

Non-Ossified space
B
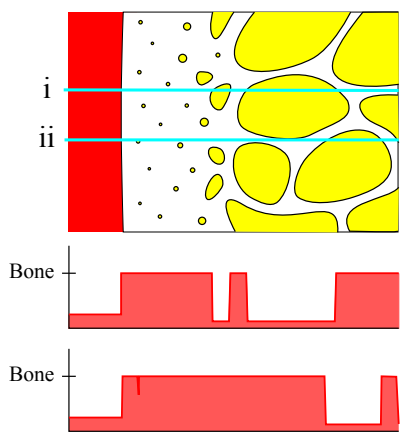

$\mathrm{C}$

ii
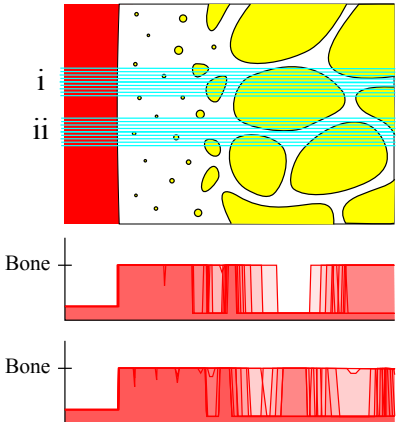

Figure 6: A two dimensional representation of the three dimensional bone microstructure and parallel profile sampling. (A) The microstructure of cortical and sub-surface trabecular bone. (B) Two sampled profiles; profile ii passes through substantially more bone than profile i despite the similar microstructure around each profile. (C) Two parallel sampled profiles, which produce much more similar aggregate density profiles.

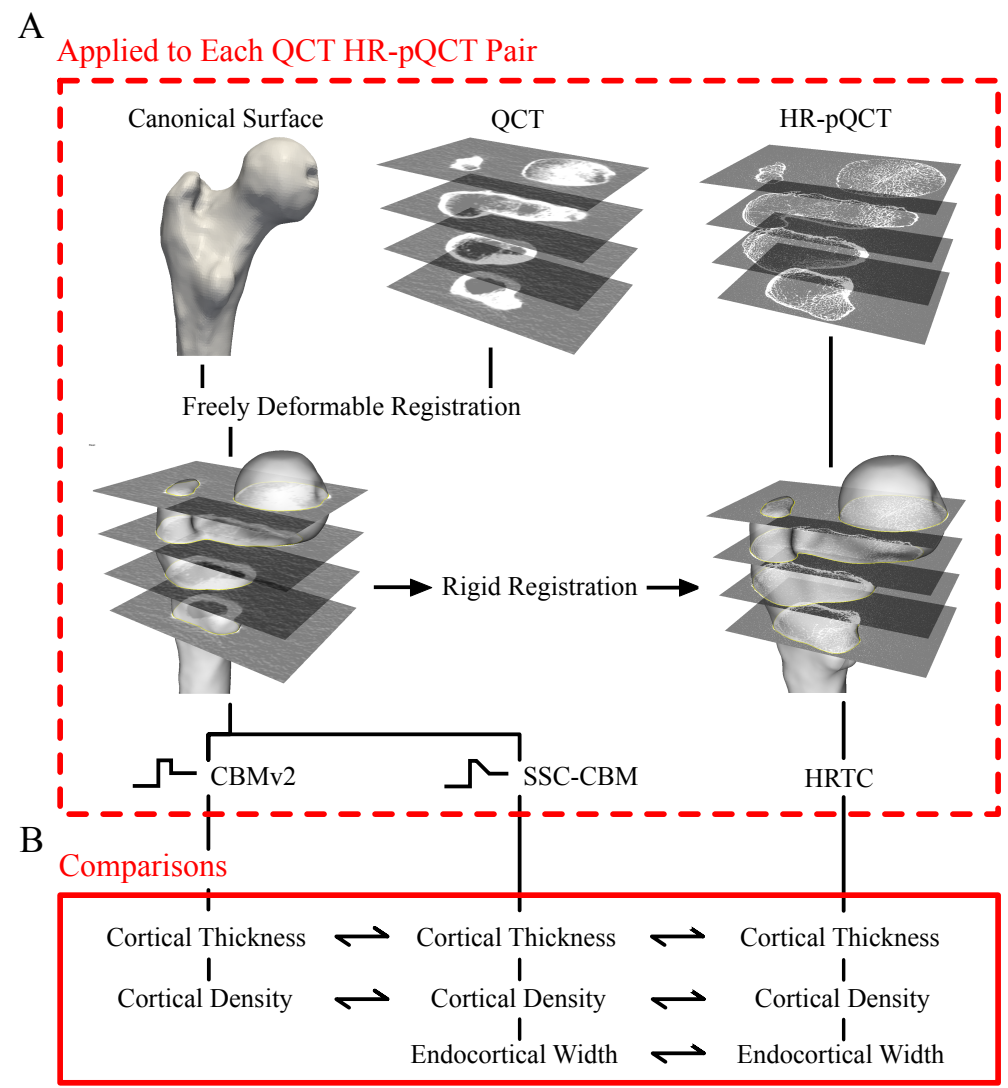

Figure 7: The pipeline used to evaluate the effectiveness of the proposed endocortical SSC-CBM implementation against both the CBMv2 method and HR-pQCT based HRTC method. (A) The registration and model fitting applied to each QCT and HR-pQCT scan pair. (B) The comparisons made between the methods.

cortical, dense cortical, endocortical and trabecular region measures produced by the two CBM methods were as-465 sessed against each other, and the HRTC validation measurements. Table 1 shows the measurement bias and precision for both CBM methods, while Figures 9 11 show confidence interval (CI) plots of CBM measurements against the corresponding HRTC measurements. These are used 470 to compare the physical features measured by the HRTC and CBM methods.

The bias and precision values recorded in Table 1 are defined through comparison with the equivalent HRTC measurements, and as in previous publications (Treece and Gee, 2015; Treece et al., 2010, 2012) these values are calculated over three different cortical thickness ranges: $0.3-1 \mathrm{~mm}, 1-3 \mathrm{~mm}$, and $3-6 \mathrm{~mm}$. The table also records the overall stability, defined as the percentage of successfully fit models out of the total number of measurement locations for each method. The CI plots show the medianbased bias and the percentile-based precision at each HRTC value in combination with the frequency distribution of the 

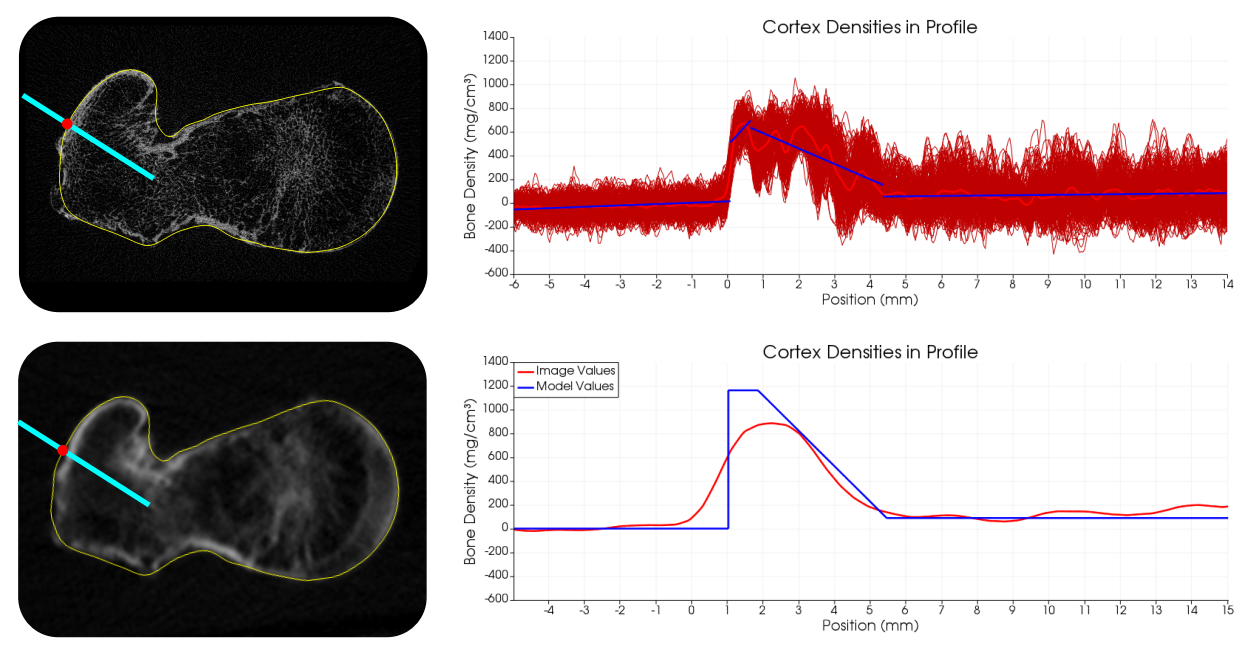

Figure 8: HR-pQCT (Top) and QCT (Bottom) profile pair with inwardly increasing intracortical porosity; the endocortical width is accurately measured.

HRTC values. Figure 9 shows the CI plots for the rect-510 angular CBMv2 method, while Figures 10 and 11 display the CI plots for the endocortical SSC-CBM method.

The rectangle model lacks any measure of the dense cortical and endocortical regions, so the bias and precision values relating to these regions are omitted from the 515 tical model were measured indirectly using Eqn 2 All other regions were directly measured from the endocortical model. The surface density for each method was also calculated across each thickness range as defined in pre-520 (Treece and Gee, 2015 : Treece et al. 2012) and included in Table 1. The cortical thickness, cBMD, tBMD and surface density CI plots for both methods are included in Figures 9 and 10, while the additional dense cortical thickness, dBMD and endocortical width measure-525 ments of the endocortical SSC-CBM method are displayed in Figure 11 .

\section{Discussion}

The values reported in Table 1 and Figures 9, 11 show that the endocortical SSC-CBM method is able to measure the thickness of the endocortical region, while broadly maintaining the quality of the cortical thickness, tBMD and MSD measurements from the CBMv2 method. The ${ }_{535}$ endocortical SSC-CBM method can measure endocortical thickness with an overall accuracy of $-0.15 \pm 0.71 \mathrm{~mm}$. The CI plots and accuracies reported in Table 1 show that the endocortical thickness measurements are accurate over the range of $1-6 \mathrm{~mm}$, but the thicknesses are underesti- ${ }_{540}$ mated for values less than $1 \mathrm{~mm}$. In addition, the endocortical thickness precisions are lower than the cortical thickness measurements made by both CBM methods. Despite this, the overall quality of the endocortical thickness measurements is notable in comparison to the cortical thick- ${ }_{545}$ ness measurements of previously validated CBM methods (Treece and Gee, 2015, Treece et al., 2012, 2010) that were used to detect statistically significant focal changes in cortical thickness in response to osteoporosis treatment therapies (Allison et al., 2015; Poole et al., 2015; Whitmarsh et al. and across populations with differing fracture risk profiles (Poole et al., 2016; Treece et al., 2015).

The rectangular CBMv2 and endocortical SSC-CBM methods both relax the single fixed density constraint applied to the model during CBM. The quality of the resulting density constraint directly affects the quality of the resulting models. Unfortunately, the constraint maps cannot be directly compared as one is of cBMD measures and the other is of dBMD measures. Instead, the cBMD values derived from the SSC-CBM endocortical model using Eqn 2b can be compared with the cBMD constraint applied to the rectangular CBMv2 model. As shown in Table 1 and Figures 9a and 10a, both methods substantially overestimate the cBMD. Despite this, the quality of the SSC-CBM cBMD measurement is improved with a visibly more linear relationship and an overall accuracy of $172 \pm 149 \mathrm{mg} / \mathrm{cm}^{3}$, compared with $249 \pm 164 \mathrm{mg} / \mathrm{cm}^{3}$ for rectangular CBMv2 method.

Eqn 2 is used to derive cortical thickness and cBMD measurements from the endocortical model. It assumes the cortical bone extends halfway along the endocortical region: it is worth considering the validity of this assumption. This is investigated in Figure 12, where Eqn 2 and the dense cortical and endocortical HRTC measurements were used to calculate derived HRTC cBMD and cortical thickness measurements; these are plotted against the measured HRTC cortical bone measurements. This shows the relationships assumed in Eqn 2 result in a slight but consistent overestimation of cBMD and cortical thickness. This does not match the overall errors in the cortical measurements, which are also impacted by the systematic over- and under-estimations in the dBMD and dense cortical thickness measurements shown in Figure 11] A closer inspection of these CI plots show that the endocor- 


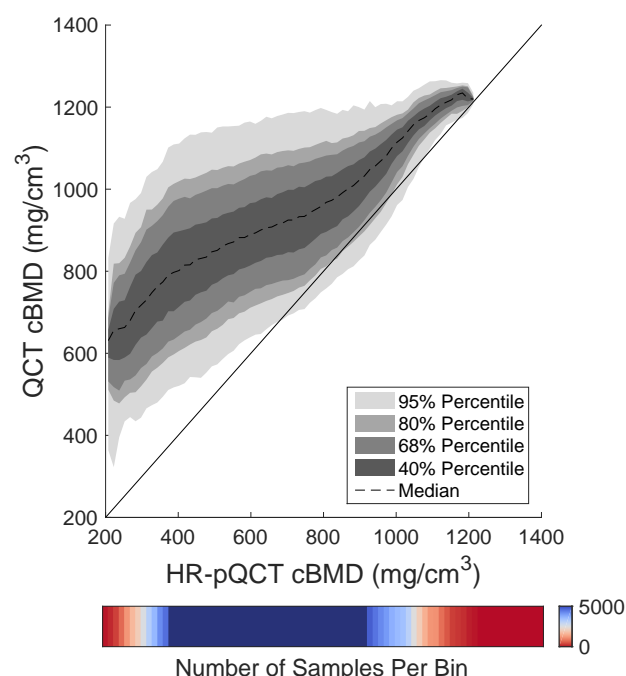

(a) Cortical density

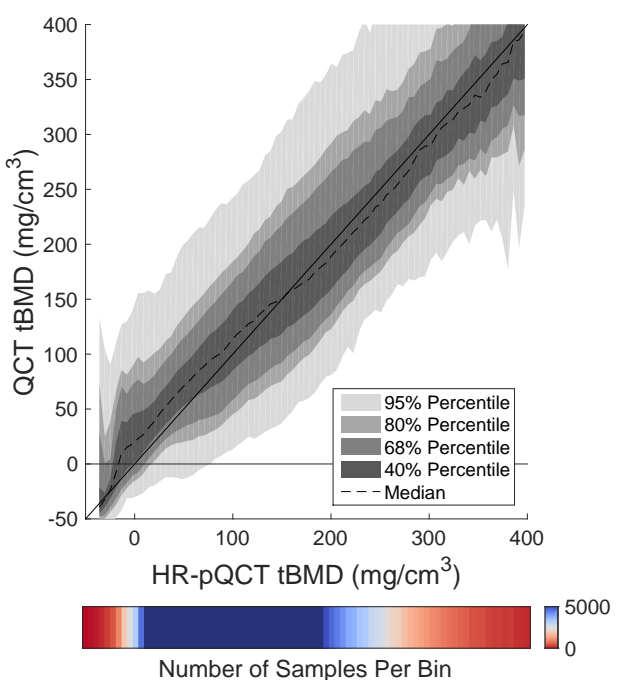

(c) Trabecular density

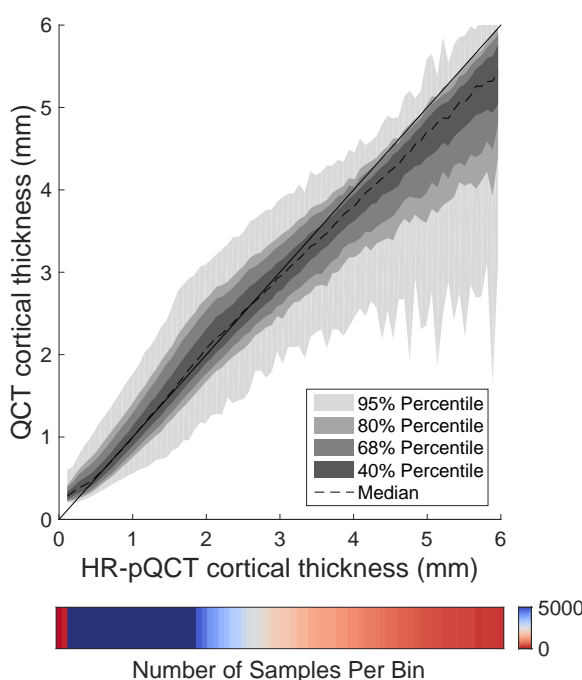

(b) Cortical thickness

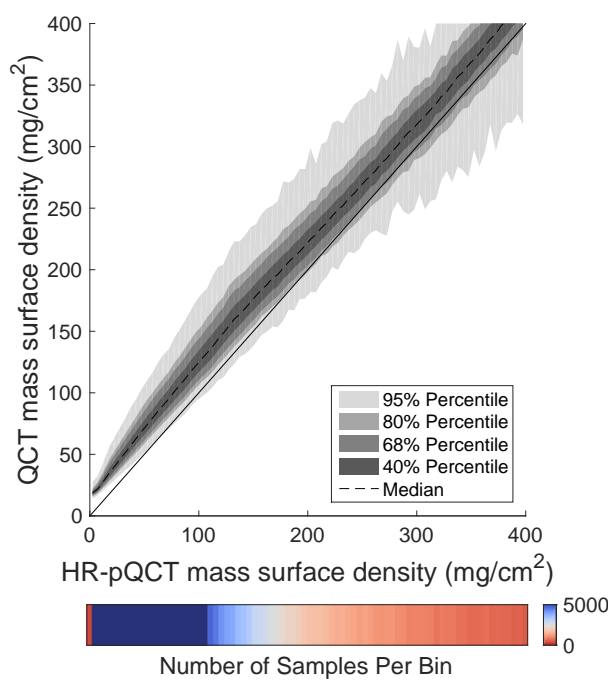

(d) Mass surface density

Figure 9: CI validation plots comparing the results of the rectangular CBMv2 method against the HRTC method. 


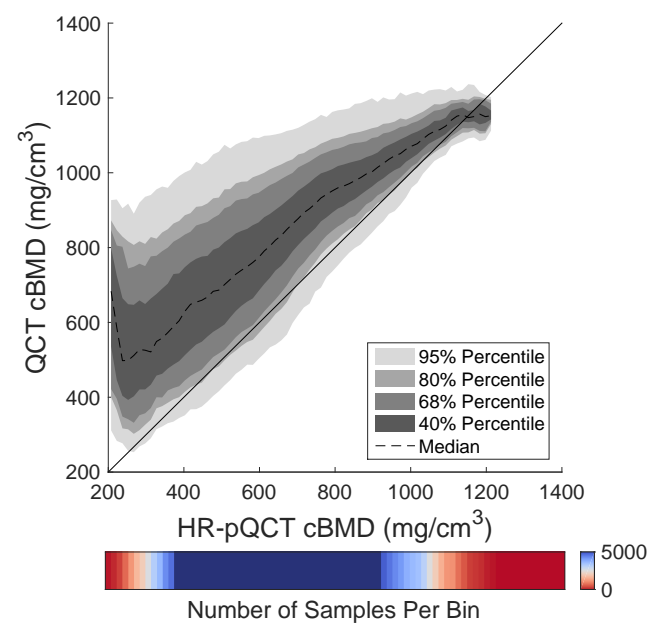

(a) Cortical density

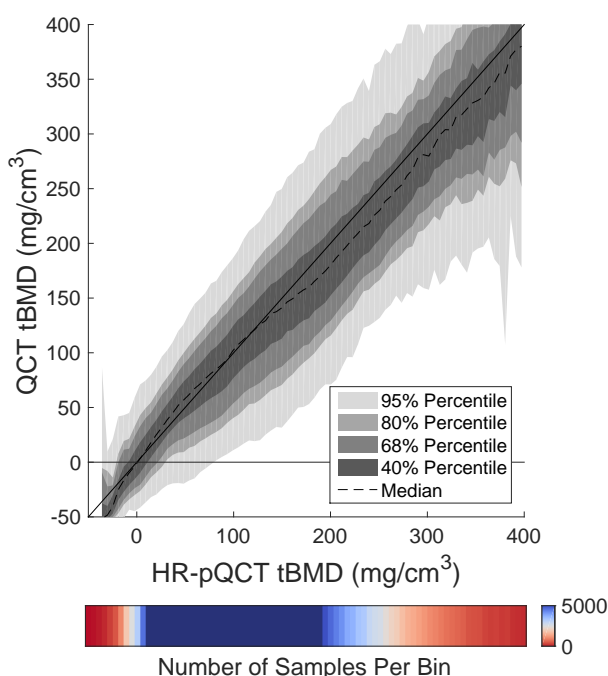

(c) Trabecular density

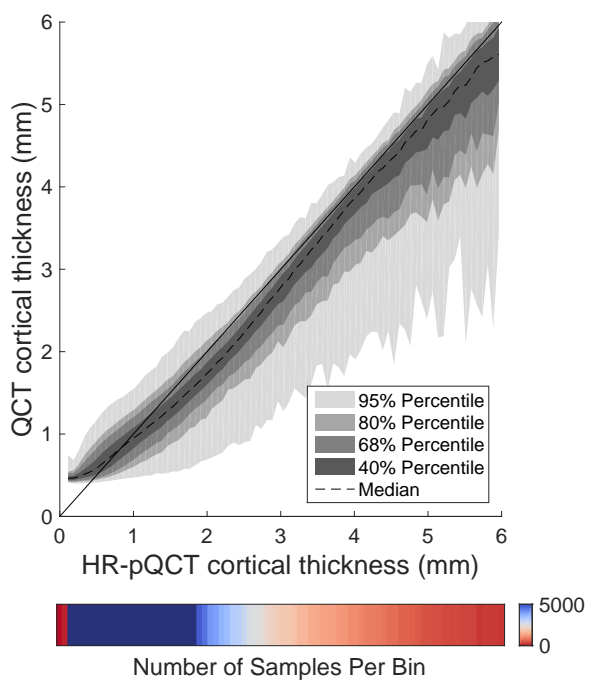

(b) Cortical thickness

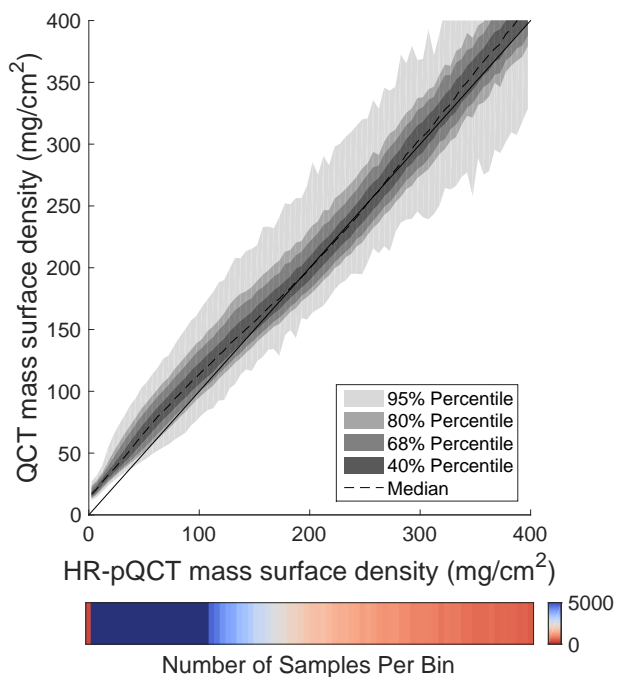

(d) Mass surface density

Figure 10: CI validation plots comparing the results of the endocortical SSC-CBM method against the HRTC method. 


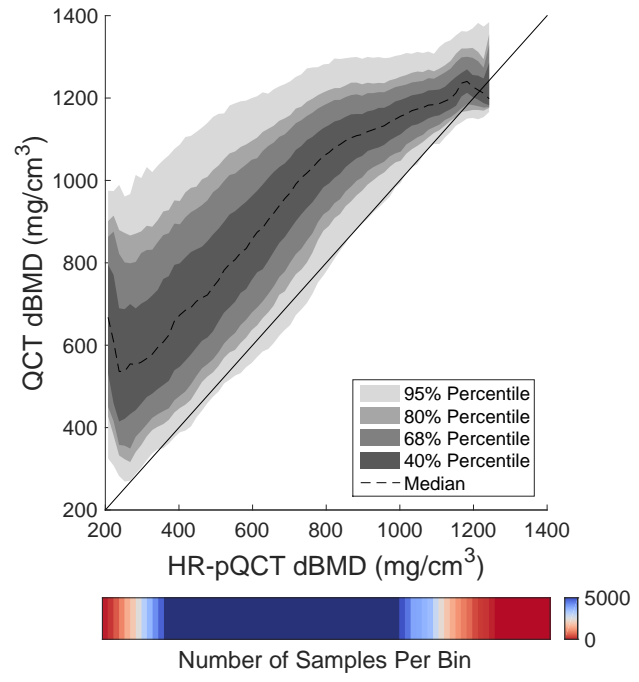

(a) Dense cortical density
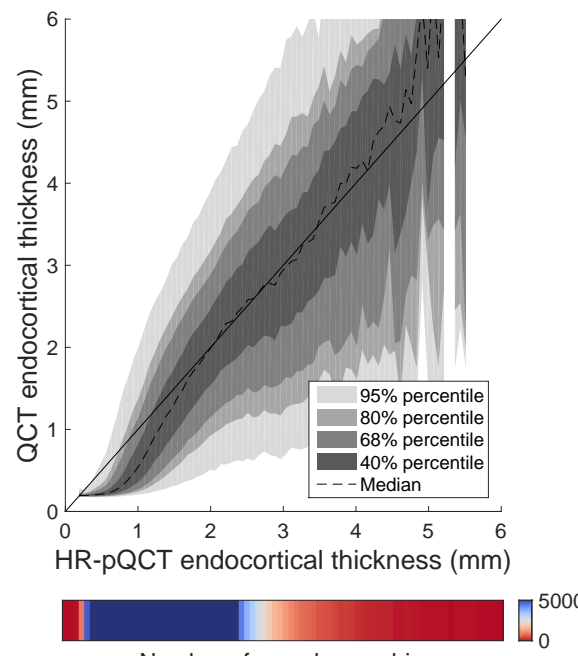

Number of samples per bin

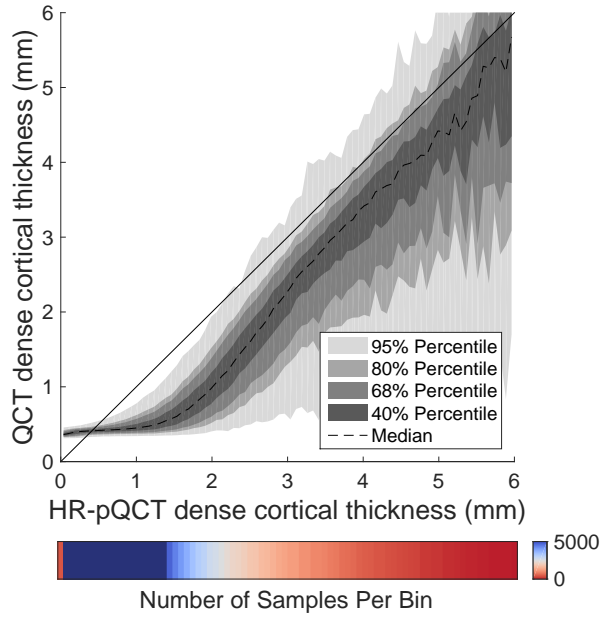

(b) Dense cortical thickness

(c) Endocortical thickness

Figure 11: CI validation plots comparing the results of the endocortical SSC-CBM method against the HRTC method, for measurements which were not previously possible from QCT. 


\begin{tabular}{|c|c|c|c|}
\hline Quantity & Thickness Ranges (mm) & $\begin{array}{l}\text { Rectangle } \\
\text { CBMv2 }\end{array}$ & $\begin{array}{l}\text { Endocortical } \\
\text { SSC-CBM }\end{array}$ \\
\hline Stability (\%) & $0.0 \leq t<6.0$ & 99.6 & 100.0 \\
\hline Cortical Thickness $(\mathrm{mm})$ & $\begin{array}{l}0.3 \leq t h_{c b}<1.0 \\
1.0 \leq t h_{c b}<3.0 \\
3.0 \leq t h_{c b}<6.0\end{array}$ & $\begin{array}{c}0.06^{*} \pm 0.23^{*} \\
0.07^{*} \pm 0.41 \\
-0.28^{*} \pm 0.60^{*}\end{array}$ & $\begin{array}{c}0.10 \pm 0.24 \\
-0.21 \pm 0.37^{*} \\
-0.34 \pm 0.61\end{array}$ \\
\hline $\begin{array}{l}\text { Cortical Density } \\
\left(\mathrm{mg} / \mathrm{cm}^{3}\right)\end{array}$ & $\begin{array}{l}0.3 \leq t h_{c b}<1.0 \\
1.0 \leq t h_{c b}<3.0 \\
3.0 \leq t h_{c b}<6.0\end{array}$ & $\begin{array}{c}321 \pm 153 \\
164^{*} \pm 127^{*} \\
129 \pm 88^{*}\end{array}$ & $\begin{array}{c}173^{*} \pm 148^{*} \\
208 \pm 152 \\
118^{*} \pm 121\end{array}$ \\
\hline $\begin{array}{l}\text { Dense Cortical Thickness } \\
(m m)\end{array}$ & $\begin{array}{l}0.3 \leq t h_{c b}<1.0 \\
1.0 \leq t h_{c b}<3.0 \\
3.0 \leq t h_{c b}<6.0\end{array}$ & $\begin{array}{l}- \\
- \\
-\end{array}$ & $\begin{array}{c}0.08 \pm 0.14 \\
-0.56 \pm 0.36 \\
-0.83 \pm 0.72\end{array}$ \\
\hline $\begin{array}{l}\text { Dense Cortical Density } \\
\left(\mathrm{mg} / \mathrm{cm}^{3}\right)\end{array}$ & $\begin{array}{l}0.3 \leq t h_{c b}<1.0 \\
1.0 \leq t h_{c b}<3.0 \\
3.0 \leq t h_{c b}<6.0\end{array}$ & $\begin{array}{l}- \\
-\end{array}$ & $\begin{array}{l}233 \pm 154 \\
306 \pm 163 \\
170 \pm 142\end{array}$ \\
\hline $\begin{array}{l}\text { Endocortical Thickness } \\
(\mathrm{mm})\end{array}$ & $\begin{array}{l}0.3 \leq t h_{c b}<1.0 \\
1.0 \leq t h_{c b}<3.0 \\
3.0 \leq t h_{c b}<6.0\end{array}$ & $\begin{array}{l}- \\
- \\
-\end{array}$ & $\begin{array}{c}-0.47 \pm 0.43 \\
0.12 \pm 0.74 \\
0.58 \pm 0.93\end{array}$ \\
\hline $\begin{array}{l}\text { Trabecular Density } \\
\left(\mathrm{mg} / \mathrm{cm}^{3}\right)\end{array}$ & $\begin{array}{l}0.3 \leq t h_{c b}<1.0 \\
1.0 \leq t h_{c b}<3.0 \\
3.0 \leq t h_{c b}<6.0\end{array}$ & $\begin{array}{l}-10^{*} \pm 42 \\
42 \pm 49 \\
80 \pm 77\end{array}$ & $\begin{array}{l}-15 \pm 42 \\
22^{*} \pm 44^{*} \\
17^{*} \pm 58^{*}\end{array}$ \\
\hline $\begin{array}{l}\text { Mass Surface Density } \\
\left(\mathrm{mg} / \mathrm{cm}^{2}\right)\end{array}$ & $\begin{array}{l}0.3 \leq t h_{c b}<1.0 \\
1.0 \leq t h_{c b}<3.0 \\
3.0 \leq t h_{c b}<6.0\end{array}$ & $\begin{array}{l}20.7 \pm 11.0^{*} \\
26.4 \pm 22.9^{*} \\
20.3 \pm 44.0^{*}\end{array}$ & $\begin{array}{l}17.2^{*} \pm 12.1 \\
12.4^{*} \pm 23.4 \\
10.1^{*} \pm 44.7\end{array}$ \\
\hline
\end{tabular}

Table 1: A comparison of the biases, precisions and overall stability of the two CBM methods assessed. The biases and precisions are calculated for each physical feature measured by each CBM method. A dash is used in place of these values for features not measured by a particular method. An * is included for bias or precision values that are significantly better than the alternative method. Significance values are calculated using a paired t-test (bias) and F-test (precision) at $p<0.001$. The stability of each method is the \% of successfully fit valid models out of the set of all measurement locations where a valid HRTC model exists.

tical SSC-CBM measurements of the dense cortical region are linearly correlated over all but the thinnest and lowest density measurements. This is where the stability of the optimiser is challenged most by the fundamental lack of information contained within the sampled density pro-580 file (Treece et al. 2010).

The endocortical SSC-CBM method can hence be used to measure the cortical thickness, cBMD, tBMD and endocortical extents. The definition of cortical thickness and tBMD measures remain unchanged from previous studies;585 namely, the cortical thickness defines the width of the cortical region, and the tBMD defines the average density of the subsurface trabecular region. The cBMD value measured by the endocortical SSC-CBM method is the mean BMD value across the cortical region as reported by the 590 HRTC method. This is in contrast to previous studies where the cBMD defined the maximum BMD value within the cortical region (Treece and Gee, 2015, Treece et al. 2012). The new cBMD value provides an aggregate measure of cumulative effect of DMB and porosity on cBMD,595 which should provide a better indication of fracture risk than just the impact of DMB (Augat and Schorlemmer, 2006: Follet et al., 2004: McCalden et al., 1993). The physical meaning of the endocortical region is less well defined: broadly it is the distance over which the uniform pores6oo within compact cortical bone transition into the larger, less uniform, marrow space of the trabecular region. Now that the method has been validated, it needs to be used on QCT data from clinical trials in order to ascertain the ability to detect statistically significant region changes in endocortical thickness and even dBMD.

The two aligned weighting functions used in the endocortical SSC-CBM method were developed in response to a systematic underestimation of cortical thickness and overestimation of tBMD for large cortical thicknesses. It is believed that these errors result from a slight decrease in BMD across the cortex due to beam hardening. The periosteal-centred weighting functions used by the rectangular CBMv2 method preferentially weight discrepancies between the modelled and the sampled density profile near the periosteal edge. This can result in the optimiser overfitting the endosteal edge location of the model to decrease the discrepancies in the cortex caused by beam hardening. This may explain some of the underestimation in cortical thickness of the rectangular CBMv2 method that occurs for cortical thicknesses greater than $3 \mathrm{~mm}$. This effect is largely counteracted by instead using the aligned weighting functions in the endocortical SSC-CBM method, as illustrated by the fairly constant bias in the SSC-CBM cortical thickness measurements greater than $2 \mathrm{~mm}$.

The initial validation of the rectangular CBMv2 method (Treece used FWHM HRpQCT measurements, rather than HRTC, though from the same paired dataset used in this study. The quality of the measurements reported in Table 1 and Figure 9 broadly agree with those reported in Treece et al. (2015), although there are subtle differences in the cortical thickness, cBMD and mass surface density measurements that stem from differences between the FWHM and HRTC 
(a)

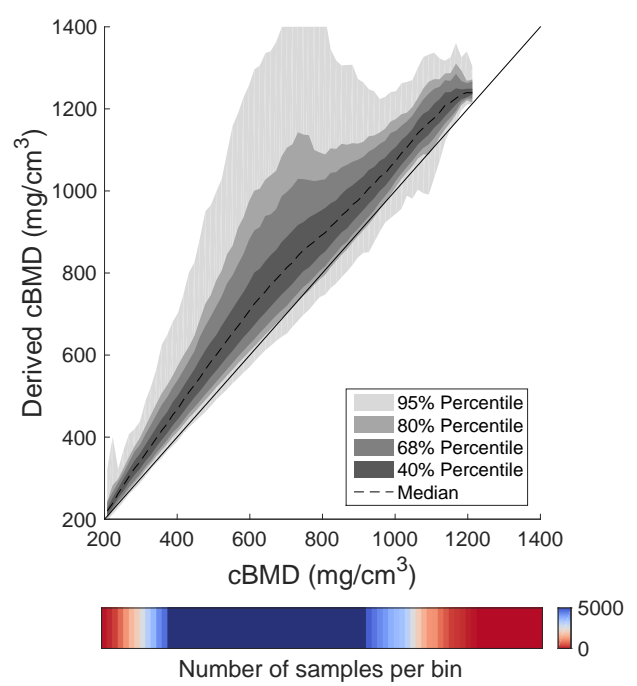

(b)

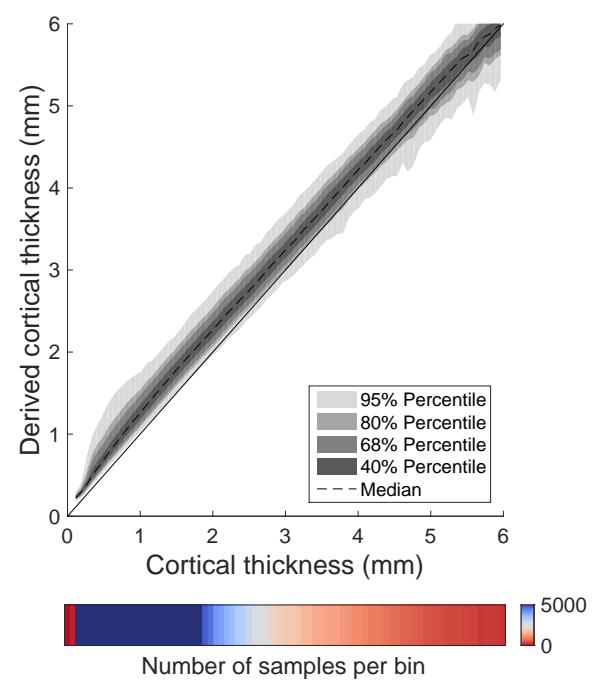

Figure 12: CI validation plots comparing direct HRTC measures of the cortical region against derived HRTC measures of the cortical region.

methods and the inclusion of two iterations of precisionbased smoothing. The FWHM method used to validate 640 previous CBM methods used the same weighting function to stabilise the optimisation process. This means its cortical thickness measurements will share any errors caused by the optimisers response to beam hardening due to unequal weighting along the profile. The FWHM method also used the peak density measured over the cortical region to define the cBMD, which is in contrast to the mean den- ${ }^{645}$ sity measurement used by the HRTC method. The mean cBMD is more clinically relevant as it incorporates the mean effects of both porosity and DMB, which both impact bone strength (Ammann and Rizzoli, 2003, Bala et al.

\section{Conclusion}

The endocortical SSC-CBM method provides a model of the cortical and subsurface trabecular region from which the cortical thickness, cBMD, tBMD and endocortical width can be measured at many locations over the proximal femur. Its ability to more accurately measure the $\mathrm{cBMD}_{655}$ at lower densities and to measure the endocortical width, while still providing similar quality cortical thickness and tBMD measurements distinguishes it from previously published QCT based techniques. The quality of the endocortical SSC-CBM and rectangular CBMv2 methods were assessed using direct comparisons between paired HR-pQCT and QCT measurements. This necessitated the development of the HRTC method, a new HR-pQCT technique, as no existing techniques were capable of providing localised cortical thickness, cBMD, tBMD and endocortical thickness measurements.

The endocortical SSC-CBM method provides sub-millimetretr endocortical thickness measurements with accuracies of $-0.47 \pm 0.43 \mathrm{~mm}, 0.12 \pm 0.74 \mathrm{~mm}$ and $0.58 \pm 0.93 \mathrm{~mm}$ over cortical thickness ranges of $0.3-1 \mathrm{~mm}, 1-3 \mathrm{~mm}$, and $3-6 \mathrm{~mm}$, and cBMD measurements down to a density of $300 \mathrm{mg} / \mathrm{cm}^{3}$, while maintaining similar quality cortical thickness and MSD measurements as CBMv2. Although this investigation shows it is possible to measure the thickness of the endocortical region from $\mathrm{QCT}$ resolution data, the clinical relevance of this measurement still needs to be established.

\section{Acknowledgements}

The authors extend their sincere thanks to Dieter Pahr, of the Vienna University of Technology, for providing the HR-pQCT and QCT scans of the ex vivo femurs. 2010).

\section{Appendix A. Appendices}

Appendix A.1. QCT Calibration

The QCT scans included a QRM-BCD calibration phantom, which contain three cores with known densities of $0 \mathrm{mg} / \mathrm{cm}^{3}, 100 \mathrm{mg} / \mathrm{cm}^{3}$ and $200 \mathrm{mg} / \mathrm{cm}^{3}$. In each scan each core was sampled in a central location across all slices along its length. These values were averaged to produce three $\mathrm{HU}$ values, which were matched with their associated density. The linear calibration coefficients for each scan were then calculated as the linear fit between the paired HU and density values. The individually calculated coefficients for each scan were then combined to give an average set of coefficients. This was only performed after the coefficients were viewed to ensure there were no outliers. A representative calibration curve is shown in FigureA.13A.

\section{Appendix A.2. HRpQCT Calibration}

The reference HR-pQCT data was captured using an XTremeCT ${ }^{4}$ scanner at $70 \mathrm{kV}$ with a pixel sizing of $0.082 \times$

\footnotetext{
${ }^{4}$ Scanco Medical AG Switzerland
} 
$0.082 \times 0.082 \mathrm{~mm}^{3}$. No calibration phantoms were included in this dataset, so an alternative calibration method had to be used. The manufacturer did provide a linear cali- ${ }^{730}$ bration curve based upon the scan settings. However due to the expected non-linearities in the HU to BMD relationship, this was not used directly. Instead, it was combined with paired HR-pQCT and QCT values extracted ${ }^{735}$ from the scans to generate a quadratic calibration curve. Specifically, the manufacturers linear HR-pQCT calibration curve and the linear QCT calibration curve were used to fabricate paired HR-pQCT and QCT HU values over the BMD $0-200 \mathrm{mg} / \mathrm{cm}^{3}$ range defined by the QRM-BCD phantom. In addition, paired HR-pQCT and QCT image values were extracted from corresponding profiles across each image pair. In each case two values were recorded: the median low, and the median high value. The median low value is the median of the first twenty percent of the profile, which should lie entirely in the surrounding saline solution. The median high value if the maximum of the QCT profile, and the median of the maximum values recorded across the multiple HR-pQCT profiles. The profiles were then filtered to remove all profiles where the cortical thickness was measured to be less than $3 \mathrm{~mm}$, as image blur might start effecting the measured QCT high median value in these cases, or where the cortex overlaps the portion of the profile used to generate the low median estimates. The remaining pairs were then combined with the fabricated data pairs before a quadratic regression line was fit as shown in Figure A.13B. This was combined with the linear QCT calibration curve to give a cubic HR-pQCT calibration curve. A representative curve is shown in Figure A.13C.

\section{References}

Allison, S.J., Poole, K.E.S., Treece, G.M., Gee, A.H., Tonkin, C., Rennie, W.J., Folland, J.P., Summers, G.D., Brooke-Wavell, K. 2015. The influence of high-impact exercise on cortical and trabecular bone mineral content and 3D distribution across the proximal ${ }_{775}$ femur in older men: A randomized controlled unilateral intervention. J. Bone Miner. Res., 30(9), 1709-1716. ISSN 1523-4681.

Ammann, P., Rizzoli, R., 2003. Bone strength and its determinants. Osteoporos. Int., 14(3), 13-18. ISSN 0937-941X.

Augat, P., Schorlemmer, S., 2006. The role of cortical bone and its microstructure in bone strength. Age Ageing, 35(Supplement 2), ii27-ii31.

Bala, Y., Farlay, D., Delmas, P.D., Meunier, P.J., Boivin, G., 2010. Time sequence of secondary mineralization and microhardness in cortical and cancellous bone from ewes. Bone, 46(4), $1204-12122_{78}$

Boivin, G., Bala, Y., Doublier, A., Farlay, D., Ste-Marie, L., Meunier, P., Delmas, P., 2008. The role of mineralization and organic matrix in the microhardness of bone tissue from controls and osteoporotic patients. Bone, 43(3), $532-538$. ISSN 8756-3282.

Burghardt, A.J., Kazakia, G.J., Sode, M., de Papp, A.E., Link, T.M., Majumdar, S., 2010. A longitudinal HR-pQCT study of alendronate treatment in postmenopausal women with low bone density: Relations among density, cortical and trabecular microarchitecture, biomechanics, and bone turnover. J. Bone Miner. Res., 25, 2558-71. ISSN 0884-0431.

Dall'Ara, E., Luisier, B., Schmidt, R., Kainberger, F., Zysset, P., Pahr, D., 2013a. A nonlinear QCT-based finite element model validation study for the human femur tested in two configurations in vitro. Bone, 52(1), 27 - 38. ISSN 8756-3282.
Dall'Ara, E., Luisier, B., Schmidt, R., Pretterklieber, M., Kainberger, F., Zysset, P., Pahr, D., 2013b. DXA predictions of human femoral mechanical properties depend on the load configuration. Med. Eng. Phys., 35(11), 1564 - 1572. ISSN 1350-4533.

Feldmar, J., Ayache, N., 1996. Rigid, affine and locally affine registration of free-form surfaces. Int. J. Comput. Vision, 18(2), 99119. ISSN 1573-1405.

Follet, H., Boivin, G., Rumelhart, C., Meunier, P., 2004. The degree of mineralization is a determinant of bone strength: a study on human calcanei. Bone, 34(5), 783 - 789. ISSN 8756-3282.

Hangartner, T.N., Gilsanz, V., 1996. Evaluation of cortical bone by computed tomography. J. Bone Miner. Res., 11(10), 1518-1525. ISSN 1523-4681.

Hangartner, T.N., Short, D.F., 2007. Accurate quantification of width and density of bone structures by computed tomography. Med. Phys., 34(10), 3777-3784.

Hildebrand, T., Rüegsegger, P., 1997. A new method for the modelindependent assessment of thickness in three-dimensional images. Journal of Microscopy 185(1), 67-75. ISSN 1365-2818.

Holzer, G., von Skrbensky, G., Holzer, L.A., Pichl, W., 2009. Hip fractures and the contribution of cortical versus trabecular bone to femoral neck strength. J. Bone Miner. Res., 24(3), 468-474. ISSN 1523-4681.

Kanis, J., McCloskey, E., Johansson, H., Cooper, C., Rizzoli, R., Reginster, J.Y., 2013. European guidance for the diagnosis and management of osteoporosis in postmenopausal women. Osteoporos. Int., 24(1), 23-57. ISSN 0937-941X.

Laib, A., Hildebrand, T., Häuselmann, H., Rüegsegger, P., 1997. Ridge number density: A new parameter for in vivo bone structure analysis. Bone, 21(6), $541-546$. ISSN 8756-3282.

Liu, X.S., Zhang, X.H., Sekhon, K.K., Adams, M.F., McMahon, D.J., Bilezikian, J.P., Shane, E., Guo, X.E., 2010. High-resolution peripheral quantitative computed tomography can assess microstructural and mechanical properties of human distal tibial bone. J. Bone Miner. Res., 25(4), 746-756. ISSN 1523-4681.

Ma, P.X., 2014. Biomaterials and regenerative medicine. Cambridge Cambridge University Press.

McCalden, R.W., McGeough, J.A., Barker, M.B., Court-Brown, C.M., 1993. Age-related changes in the tensile properties of cortical bone. The relative importance of changes in porosity, mineralization, and microstructure. J. Bone Joint Surg., 75(8), 11931205. ISSN 0021-9355.

Moré, J.J., 1978. The Levenberg-Marquardt algorithm: Implementation and theory. In G.A. Watson, editor, Numerical Analysis: Proceedings of the Biennial Conference Held at Dundee, June 28July 1, 1977, 105-116. Springer Berlin Heidelberg, Berlin, Heidelberg. ISBN 978-3-540-35972-2

Ohnaru, K., Sone, T., Tanaka, K., Akagi, K., Ju, Y.I., Choi, H.J., Tomomitsu, T., Fukunaga, M., 2013. Hip structural analysis: a comparison of DXA with CT in postmenopausal japanese women. SpringerPlus, 2(1), 331. ISSN 2193-1801.

Pakdel, A., Robert, N., Fialkov, J., Maloul, A., Whyne, C., 2012. Generalized method for computation of true thickness and x-ray intensity information in highly blurred sub-millimeter bone features in clinical CT images. Phys. Med. Biol., 57(23), 8099.

Poole, K., Skingle1, L., Gee, A., Turmezei, T., Johannesdottir, F., Blesic, K., Rose, C., Vindlacheruvu, M., Donell, S., Vaculiz, J., Dungl, P., Horak, M., Stepan, J., Reeve, J., Treece, G., 2016. Focal osteoporotic defects play a key role in hip fracture. Bone, 94, 124-134. ISSN 8756-3282.

Poole, K.E., Treece, G.M., Gee, A.H., Brown, J.P., McClung, M.R., Wang, A., Libanati, C., 2015. Denosumab rapidly increases cortical bone in key locations of the femur: A 3D bone mapping study in women with osteoporosis. J. Bone Miner. Res., 30(1), 46-54. ISSN 1523-4681.

Poole, K.E.S., Treece, G.M., Mayhew, P.M., Vaculík, J., Dungl, P., Horák, M., Štěpán, J.J., Gee, A.H., 2012. Cortical thickness mapping to identify focal osteoporosis in patients with hip fracture. PLoS ONE, 7(6), e38466.

Seeman, E., Delmas, P.D., 2006. Bone quality - the material and structural basis of bone strength and fragility. New Engl. J. Med., 
(a)

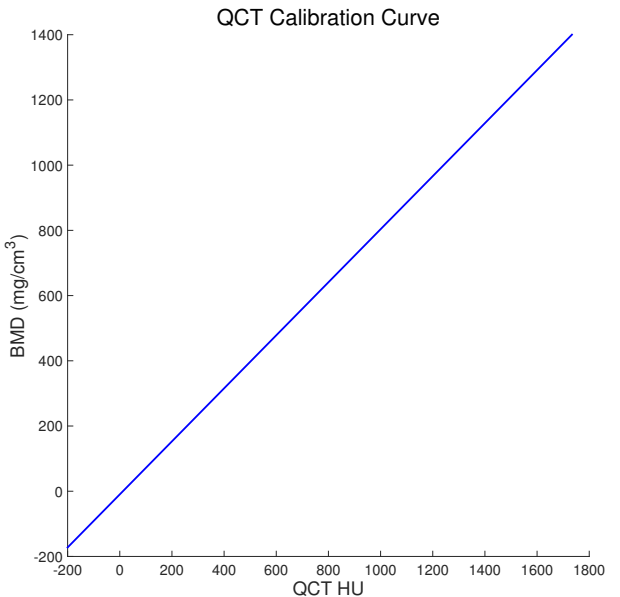

(c)

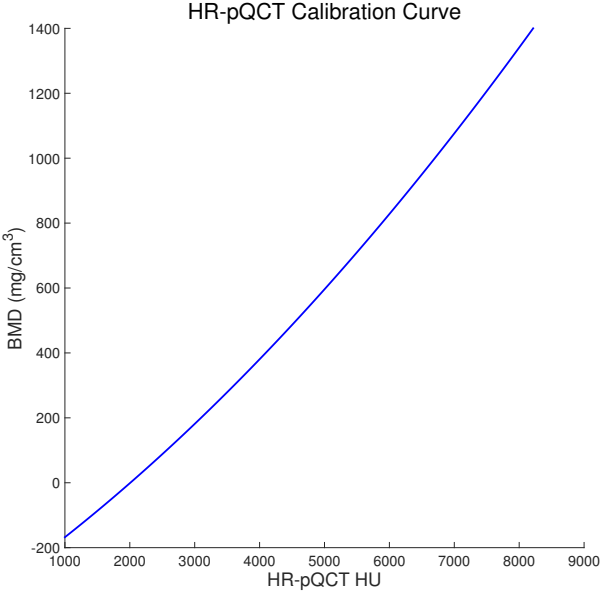

(b)

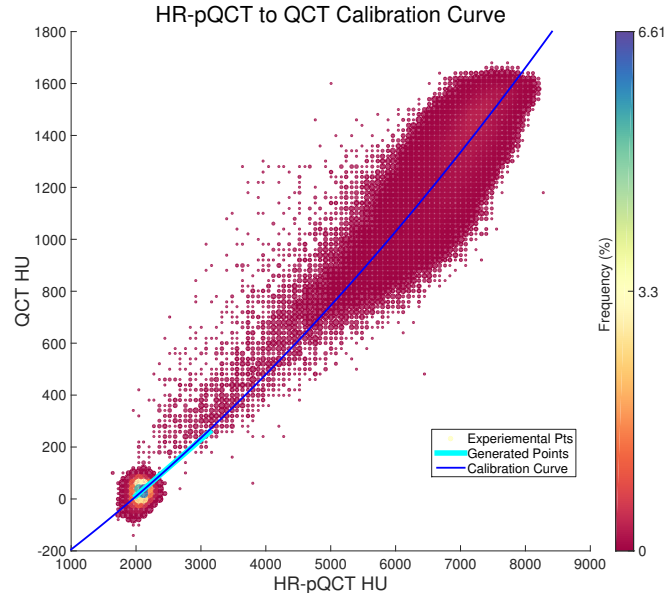

Figure A.13: (A) The linear calibration applied to QCT scans to convert QCT HU units into BMD values. (B) The quadratic calibration curve and the data from which this was generated, used to convert HR-pQCT HU into QCT HU units. (C) The complete quadratic calibration curve applied to HR-pQCT scans to convert HR-pQCT HU units into BMD values. 
$354(21), 2250-2261$

Streekstra, G.J., Strackee, S.D., Maas, M., ter Wee, R., Venema, H.W., 2007. Model-based cartilage thickness measurement in the submillimeter range. Med. Phys., 34(9), 3562-3570.

Treece, G., Gee, A., 2015. Independent measurement of femoral cortical thickness and cortical bone density using clinical CT. Med. Image Anal., 20(1), 249 - 264. ISSN 1361-8415

Treece, G., Gee, A., Mayhew, P., Poole, K., 2010. High resolution cortical bone thickness measurement from clinical CT data. Med. Image Anal., 14(3), 276 - 290. ISSN 1361-8415.

Treece, G., Poole, K., Gee, A., 2012. Imaging the femoral cortex: Thickness, density and mass from clinical CT. Med Image Anal., 16(5), 952 - 965. ISSN 1361-8415.

Treece, G.M., Gee, A.H., Tonkin, C., Ewing, S.K., Cawthon, P.M., Black, D.M., Poole, K.E., 2015. Predicting hip fracture type with cortical bone mapping (CBM) in the osteoporotic fractures in men (MrOS) study. J. Bone Miner. Res., 30(11), 2067-2077. ISSN 1523-4681.

Verhulp, E., van Rietbergen, B., Huiskes, R., 2008. Load distribution in the healthy and osteoporotic human proximal femur during a fall to the side. Bone, 42(1), 30 - 35. ISSN 8756-3282.

Whitmarsh, T., Treece, G., Gee, A., Bolognese, M., Brown, J., Goemaere, S., Grauer, A., Hanley, D., Mautalen, C., Recknor, C., Yang, Y.C., Libanati, C., Poole, K., 2014. Romosozumab and teriparatide effects on vertebral cortical mass, thickness and density in postmenopausal women with low bone mineral density (BMD). In Proceedings of the American Society for Bone and Mineral Research. ASBMR, Houston, USA.

Whitmarsh, T., Treece, G.M., Gee, A.H., Poole, K.E.S., 2015 Mapping bone changes at the proximal femoral cortex of postmenopausal women in response to alendronate and teriparatide alone, combined or sequentially. J. Bone Miner. Res., 30(7), 13091318. ISSN 1523-4681.

Whitmarsh, T., Treece, G.M., Gee, A.H., Poole, K.E.S., 2016. The effects on the femoral cortex of a 24 month treatment compared to an 18 month treatment with teriparatide: A multi-trial retrospective analysis. PLoS ONE, 11(2), 1-9.

World Health Organisation, 2007. WHO Scientific Group on the Assessment of Osteoporosis at Primary Health Care Level, Summary Meeting Report. World Health Organisation, Geneva.

Yang, L., Burton, A.C., Bradburn, M., Nielson, C.M., Orwoll, E.S., Eastell, R., for the Osteoporotic Fractures in Men (MrOS) Study Group, 2012. Distribution of bone density in the proximal femur and its association with hip fracture risk in older men: The osteoporotic fractures in men (MrOS) study. J. Bone Miner. Res., 27(11), 2314-2324. ISSN 1523-4681.

Zebaze, R., Ghasem-Zadeh, A., Mbala, A., Seeman, E., 2013. A new method of segmentation of compact-appearing, transitional and trabecular compartments and quantification of cortical porosity from high resolution peripheral quantitative computed tomographic images. Bone, 54(1), 8 - 20. ISSN 8756-3282.

Zebaze, R.M., Libanati, C., Austin, M., Ghasem-Zadeh, A., Hanley, D.A., Zanchetta, J.R., Thomas, T., Boutroy, S., Bogado, C.E., Bilezikian, J.P., Seeman, E., 2014. Differing effects of denosumab and alendronate on cortical and trabecular bone. Bone, 59(0), 173 - 179. ISSN 8756-3282. 